\title{
Bit Error Rate Analysis for OFDM Systems for MRC Receivers in Nakagami- m Fading Channel Generated by Using Rayleigh and Ricean Fading
}

\author{
Vijit Gupta, Sahil Chaudhary and Ankit Jain
}

\begin{abstract}
Error rates of orthogonal frequency division multiplexing (OFDM) signals are considered for system using multichannel reception with maximum ratio combining (MRC) receivers in Nakagami-m fading generated by sum of sinusoidal method using Rayleigh and Ricean channel. The paper also discusses the effect of frequency offsets, nakagami-m parameter $\mathrm{m}$ on OFDM systems. Also effect of frequency offset on signal to noise plus interference ratio (SINR) for different SNR values is discussed. A closed form expression for probability of error is derived and theoretical and simulated results are compared for various receivers, frequency offsets.
\end{abstract}

\section{INTRODUCTION}

Due to its robustness against time dispersion in multipath fading channels orthogonal frequency division multiplexing is an important wide band transmission scheme for modern communication systems[1]. In it parallel frequency channels are used to transmit the data which are modulated.This is believed to be used as a standard transmission techniques for various services like fourth generation mobile communication systems, digital terrestrial TV broadcasting etc.Another advantage of ofdm is its relatively simple equalizer at the receiver and its suitability for transmission over multipath fading channel .Different fading models are present that have been used to study wireless communication systems. Most important are like Rayleigh and ricean .When there is no los component between mobile station and base station Rayleigh fading comes to picture when there is a dominant stationary signal component present ,fading envelope distribution is ricean[2]. Recently nakagami distribution has been of great interest because nakagami fading could represent various fading condition in wireless channel $[2,3,4,5]$. So analysis of nakagami channel is very important. Analysis of OFDM systems in Rayleigh and ricean fading is done in [6]. Maximum ratio combining (MRC) is a special form of general diversity combining, by which multiple replicas of the same information-bearing signal received over different diversity branches are combined so as to maximize the instantaneous SNR at the combiner output [7].Firstly we mathematically calculated the probability of error for maximum ratio combining receivers for OFDM systems in nakagami fading later compared the theoretical result with the simulated results. The paper is divided into various sections. In first part we have given brief introduction of orthogonal frequency division multiplexing and various common fading channels, in second section we have done mathematical analysis of MRC in nakagami fading in OFDM systems. Later in the simulation results section we have compared our simulated results with the theoretical results derived in earlier section

\section{SYSTEM MODEL}

Figure 1 shows a typical OFDM transmitter and receivers described in [8]. In this paper we have modulated the incoming data by using BPSK (binary phase shift keying scheme).The incoming serial bits are converted into parallel streams by using serial to parallel convertor block. There after these parallel streams are subjected to IDFT block. Then due to overlapping of the data at the receiver cyclic prefix is inserted whose duration should be greater then the maximum delay spread. Then before transmitting the data in the channel we have converted the digital data to analog one by passing it through digital to analog convertor. Then the data is transmitted to the channel at the receiver side firstly the data is converted again to digital domain by passing it to the analog to digital convertor then cyclic prefix are removed then we do DFT and then parallel to serial block . 


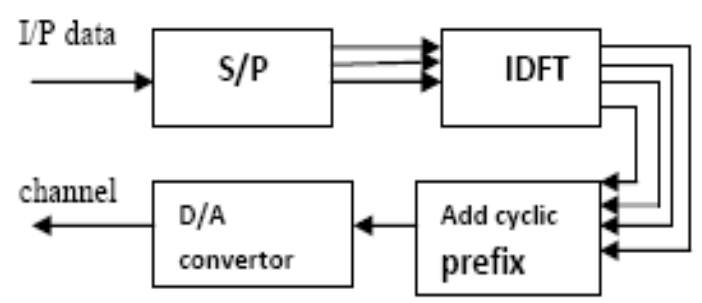

Fig(1)OFDM transmitter

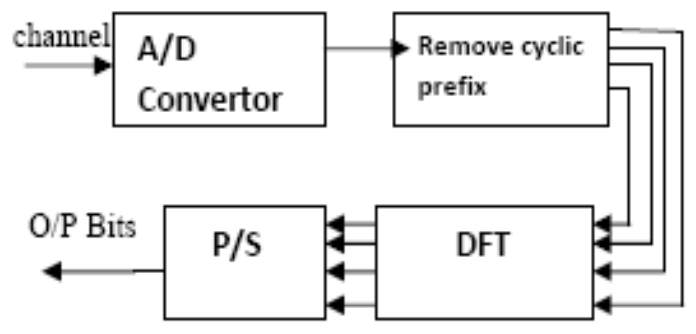

Fig (2).OFDM receiver

\section{MATHEMATICAL ANALYSIS}

The IFFT output at the transmitter from [8], can be written as:

$$
x_{n}=\frac{1}{N} \sum_{k=-K}^{K} X k e^{2 \pi j n k / N}
$$

Where $\mathrm{n}=0,1,2 \ldots \ldots, \mathrm{N}-1$, and $\mathrm{N} \geq 2 \mathrm{~K}+1$, where $\mathrm{K}$ is the number of subcarriers and $\mathrm{N}$ is the period of IFFT.

After passing through the channel (Nakagami in this case) the output can be expressed as in [8],

$$
y_{n}=\frac{1}{N}\left[\sum_{k=-K}^{K} X_{k} H_{k} e^{2 \pi j n(k+\varepsilon) / N}\right]+w_{n}
$$

Where $\mathrm{n}=0,1,2 \ldots \ldots, \mathrm{N}-1$, where $H_{k}$ is the channel transfer function at the frequency of the kth subcarrier, $E$ is the relative frequency offset, $w_{n}$ is the additive white Gaussian noise. Output of the DFT modulator can be written as

$$
Y_{k}=\sum_{n=0}^{N-1}\left\{\frac{1}{N}\left[\sum_{n=-K}^{K} H_{k} X_{k} e^{2 \pi j n(k+\varepsilon) / N}\right]+w_{n}\right\} e^{-2 \pi j n k / N}
$$

$$
\begin{gathered}
Y_{k}=\left(X_{k} H_{k}\right)\left\{\frac{\sin (\pi \varepsilon)}{N \sin (\pi \varepsilon / N)}\right\} e^{j \pi \varepsilon(N-1) / N}+I_{k}+W_{k} \\
I_{k}=\sum_{\substack{l=0 \\
l \neq k}}^{N-1} \frac{1}{N} X_{l} H_{l}\left[\frac{\sin \pi(l+\varepsilon-k)}{\sin \pi \frac{(+\varepsilon-k)}{N}}\right] e^{j \pi(N-1)\left(\frac{l+\varepsilon-k)}{N}(2)\right.}
\end{gathered}
$$

Using the moment generating technique to find the average probability of error for BPSK modulation in nakagami fading channel is given by [9]

$$
P_{b}=\frac{1}{\pi} \int_{0}^{\pi / 2}\left(1+\frac{\operatorname{SINR}}{m\left(\sin ^{2} \phi\right)}\right)^{-m} d \phi
$$

Where SINR is average signal to interference plus noise ratio .then for $\mathrm{z}$ receiver for MRC uncorrelated channels we have

$$
P_{b}=\frac{1}{\pi} \int_{0}^{\pi / 2}\left[\left(1+\frac{\operatorname{SINR}}{m\left(\sin ^{2} \phi\right)}\right)^{-m}\right]^{L} d \phi
$$

Where $\mathrm{L}$ is the number of receivers. Now we will calculate SINR, as described already SINR is average signal to interference plus noise ratio.

$$
\begin{aligned}
& \operatorname{SINR}=\frac{s^{2}}{\sigma_{n}^{2}+\sigma_{I}^{2}} \\
& S=\left(X_{k} H_{k}\right)\{\sin (\pi \varepsilon) / N \sin (\pi \varepsilon / N)\}
\end{aligned}
$$

For $\mathrm{N} \gg \pi \varepsilon$ and taking an approximation

$$
\begin{aligned}
& E\left[X_{k}\right]=0 \\
& S^{2}=X^{2} E\left\{\left|H_{k}\right|^{2}\right\} \sin (\pi \varepsilon)^{2} /(\pi \varepsilon)^{2}
\end{aligned}
$$

Value of $E\left\{\left.H_{k}\right|^{2}\right\}$ is calculated in the next section (eq.5) which comes out to be $\Omega$, in this paper we have taken $\Omega=1$.so the above equation reduces to

$$
S^{2}=\frac{X^{2}}{(\pi \varepsilon)^{2}}(\sin (\pi \varepsilon))^{2}
$$


Here we have also assumed that $E\left[X_{k}\right]=0$ so by this provision we have

$$
\sigma_{I}^{2}=E\left\{\left|I_{k}\right|^{2}\right\}
$$

Now considering equation (2) two cases arises:

If (1-k) is even, then $\sin \pi(l-\varepsilon-k)=-\sin (\pi \varepsilon)$

If (l-k) is even, then $\sin \pi(l-\varepsilon-k)=\sin (\pi \varepsilon)$ for both the cases we have

$$
E\left\{\left.I_{k}\right|^{2}\right\}=X^{2} E\left\{\left.H_{k}\right|^{2}\right\} \sin ^{2}(\pi \varepsilon) \sum_{l=0}^{N-1} \frac{1}{\left\{N \sin \frac{(l-k-\varepsilon)}{N}\right\}^{2}}
$$

Here we are taking period of IFFT $=\mathrm{N}=64$

$$
E\left\{\left|H_{k}\right|^{2}\right\}=\Omega=1
$$

And substituting $\mathrm{k}=0$ and $\varepsilon=0.05$

$$
\sigma_{I}^{2}=X^{2} \sin (\pi \varepsilon)^{2}(0.3349)
$$

Now defining $\frac{X^{2}}{\sigma_{n}^{2}}=S N R$

SINR can be expressed as

$$
\operatorname{SINR}=\frac{\frac{S^{2}}{\sigma_{n}^{2}}}{1+\frac{\sigma_{I}^{2}}{\sigma_{n}^{2}}}
$$

After substituting the values, we get

$$
\operatorname{SINR}=\frac{\operatorname{SNR} \frac{\{\sin (\pi \varepsilon)\}^{2}}{(\pi \varepsilon)^{2}}}{\operatorname{SNR}\{\sin (\pi \varepsilon)\}^{2}(0.3349)+1}
$$

Substituting value of SINR in (3) we get final expression as

$$
P_{b}=\frac{1}{\pi} \int_{0}^{\pi / 2}\left[\left(\left[1+\frac{S N R \frac{\{\sin (\pi \varepsilon)\}^{2}}{(\pi \varepsilon)^{2}}}{m\left(\sin ^{2} \phi\right)\left(\operatorname{SNR}\{\sin (\pi \varepsilon)\}^{2}(0.3349)+1\right)}\right]\right)^{-m}\right]^{L} d \phi
$$

\section{CALCULATION OF SECOND MOMENT OF NAKAGAMI FADING}

The PDF of nakagami distribution is generally defined as

$$
\begin{aligned}
& F(x)=\frac{2 m^{m} x^{2 m-1}}{\Omega^{m \Gamma(m)}} \exp \left(-\frac{m x^{2}}{\Omega}\right) \\
& \mathrm{m} \geq 0.5, \mathrm{x} \geq 0
\end{aligned}
$$

Now let us try to calculate the kth moment nakagami pdf by definition-

$$
E\left(X^{k}\right)=\int_{0}^{\infty} F(X) X^{k} d x
$$

THE RHS becomes

$$
\int_{0}^{\infty} \frac{2 m^{m} x^{2 m-1+k}}{\Omega^{m \Gamma(m)}} \exp \left(-\frac{m x^{2}}{\Omega}\right) d x
$$

As we know that from [6,eqn. 3.681].

$$
\int_{0}^{x} x^{\nu-1} \exp \left(-\mu x^{2}\right) d x=\frac{\mu\left(-\frac{v}{z}\right)}{z} \Gamma\left(\frac{v}{2}\right)
$$

Comparing the above two equations-

$\mu=\mathrm{m} / \Omega, \mathrm{z}=2, \mathrm{v}=2 \mathrm{~m}+\mathrm{k}$

After slight calculation we get

$$
E\left(X^{k}\right)=\left(\frac{\Omega}{m}\right)^{\frac{k}{2}} \Gamma\left(m+\frac{k}{2}\right) \frac{1}{\Gamma m}
$$

For second moment we have $\mathrm{K}=2$, We get $E\left(X^{2}\right)=\Omega$, where $X$ is nakagami PDF, using symbol $\mathrm{H}$ in place of $\mathrm{X}$ we get $E\left(H^{2}\right)=\Omega$

\section{NAKAGAMI FADING GENERATION}

For simulation purpose we have generated the nakagami fading by using sum of sinusoidal using Rayleigh and ricean fading as given in [2] Eqn. 6. The received signal for Rayleigh can be expressed as

$$
R_{\text {nakagami }}=R_{\text {ray }} e^{(1-m)}+R_{\text {rice }}\left(1-e^{(1-m)}\right)
$$

Where $R_{\text {ray }}$ and $R_{\text {rice }}$ are envelops of Rayleigh and ricean channels respectively. Their method of generation is given in [2]. By adopting sum of sinusoidal approach for received signal generation 
and expressing it in inphase and quadrature form for both Rayleigh and ricean fading.

$$
R(t)=\sqrt{[I(t)]^{2}+[Q(t)]^{2}}
$$

Where $\mathrm{I}(\mathrm{t})$ and $\mathrm{Q}(\mathrm{t})$ are the inphase and quadrature components[2].

\section{SIMULATION RESULTS}

In this section, both computer simulations and a theoretical analysis are carried out to investigate the BER performance of an ofdm system in Nakagami- $m$ fading channel for various receivers and frequency offsets values. Data bits were taken as 10000, SNR in $\mathrm{dB}$ is taken from 0 to 12 , guard band interval is taken to 7 .

In figure 3,4 BER vs. frequency offset is shown for different SNR for 2 and 4 receivers respectively. We see that for a given frequency offset, BER decreases as SNR is increases.

\section{TABLE I}

\begin{tabular}{|l|l|l|l|}
\hline & $\begin{array}{l}\text { Freq. offset } \\
(0.05)\end{array}$ & $\begin{array}{l}\text { Freq. offset } \\
(0.15)\end{array}$ & $\begin{array}{l}\text { Freq.offset } \\
(0.25)\end{array}$ \\
\hline BER(SNR=4) & 0.0102 & 0.0152 & 0.0269 \\
\hline BER(SNR=6) & 0.0039 & 0.0072 & 0.0159 \\
\hline
\end{tabular}

BER for different frequency offset for 2 receivers.

TABLE II

\begin{tabular}{|l|l|l|l|}
\hline & $\begin{array}{l}\text { Freq. offset } \\
(0.05)\end{array}$ & $\begin{array}{l}\text { Freq. offset } \\
(0.15)\end{array}$ & $\begin{array}{l}\text { Freq. offset } \\
(0.25)\end{array}$ \\
\hline BER(SNR=4) & 0.0004 & 0.0009 & 0.0027 \\
\hline BER(SNR=4) & 0.0001 & 0.0002 & 0.0010 \\
\hline
\end{tabular}

BER for different frequency offset for 2 receivers.

In figures 5 , BER vs. SNR is plotted for frequency offset 0.05 and 0.15 .
TABLE III

\begin{tabular}{|l|l|l|l|l|}
\hline & $\begin{array}{l}\text { SNR } \\
(0 \mathrm{~dB})\end{array}$ & $\begin{array}{l}\text { SNR } \\
(4 \mathrm{~dB})\end{array}$ & $\begin{array}{l}\text { SNR } \\
(8 \mathrm{~dB})\end{array}$ & $\begin{array}{l}\text { SNR } \\
(12 \mathrm{~dB})\end{array}$ \\
\hline $\begin{array}{l}\text { BER(freq. } \\
\text { off.=0.05) }\end{array}$ & 0.0473 & 0.0102 & 0.0014 & 0.0002 \\
\hline $\begin{array}{l}\text { BER(freq. } \\
\text { off.=0.15) }\end{array}$ & 0.0558 & 0.0152 & 0.0034 & 0.0009 \\
\hline
\end{tabular}

BER vs. SNR is plotted for frequency offset 0.05 and 0.15 .

In figure 6, signal to noise plus interference ratio vs. frequency offset is plotted for different SNR. We see that for a given SNR, SINR decreases with increasing frequency offset, where as for a given frequency offset value of SINR increases as the SNR increases.

TABLE IV

\begin{tabular}{|l|l|l|l|}
\hline & $\begin{array}{l}\text { Freq.offset } \\
(0.05)\end{array}$ & $\begin{array}{l}\text { Freq.offset } \\
(0.15)\end{array}$ & $\begin{array}{l}\text { Freq.offset } \\
(0.3)\end{array}$ \\
\hline $\begin{array}{l}\text { SINR } \\
(\mathrm{SNR}=4 \mathrm{~dB})\end{array}$ & 2.4410 & 1.9869 & 1.1936 \\
\hline $\begin{array}{l}\text { SINR } \\
(\mathrm{SNR}=8 \mathrm{~dB})\end{array}$ & 5.9502 & 4.079 & 1.9509 \\
\hline $\begin{array}{l}\text { SINR } \\
(\mathrm{SNR}=10 \mathrm{~dB})\end{array}$ & 9.1668 & 5.4911 & 2.3084 \\
\hline
\end{tabular}

Signal to noise plus interference ratio vs. frequency offset

In figure 7, BER vs. SNR for different values of nakagami fading parameter $\mathrm{m}$ is shown. 
TABLE V

\begin{tabular}{|l|l|l|l|l|}
\hline & $\begin{array}{l}\text { SNR } \\
(0 \mathrm{~dB})\end{array}$ & $\begin{array}{l}\text { SNR } \\
(4 \mathrm{~dB})\end{array}$ & $\begin{array}{l}\text { SNR } \\
(8 \mathrm{~dB})\end{array}$ & $\begin{array}{l}\text { SNR } \\
(10 \mathrm{~dB})\end{array}$ \\
\hline BER(m=0.05) & 0.0929 & 0.0445 & 0.0198 & 0.0087 \\
\hline BER(m=1.5) & 0.0473 & 0.0102 & 0.0014 & 0.0001 \\
\hline BER(m=2.0) & 0.0413 & 0.0071 & 0.0006 & 0.00005 \\
\hline
\end{tabular}

BER vs. SNR for different values of nakagami fading parameter $\mathrm{m}$

In fig. 8, BER vs. SNR is shown for different receivers 3 and 4 respectively. We see from these figures that theoretical and simulated results are closely matched. We see that BER decreases as the number of receivers are increased.

TABLE VI

\begin{tabular}{|l|l|l|l|l|}
\hline & $\begin{array}{l}\text { SNR } \\
(0 \mathrm{~dB})\end{array}$ & $\begin{array}{l}\text { SNR } \\
(4 \mathrm{~dB})\end{array}$ & $\begin{array}{l}\text { SNR } \\
(8 \mathrm{~dB})\end{array}$ & $\begin{array}{l}\text { SNR } \\
(10 \mathrm{~dB})\end{array}$ \\
\hline $\begin{array}{l}\text { BER(3 } \\
\text { receivers) }\end{array}$ & 0.01900 & 0.00202 & 0.000104 & 0.00002016 \\
\hline $\begin{array}{l}\text { BER(4 } \\
\text { receivers })\end{array}$ & 0.00794 & 0.000418 & 0.0000082 & 0.00000093 \\
\hline
\end{tabular}

BER vs. SNR is shown for 3 and 4 receivers.

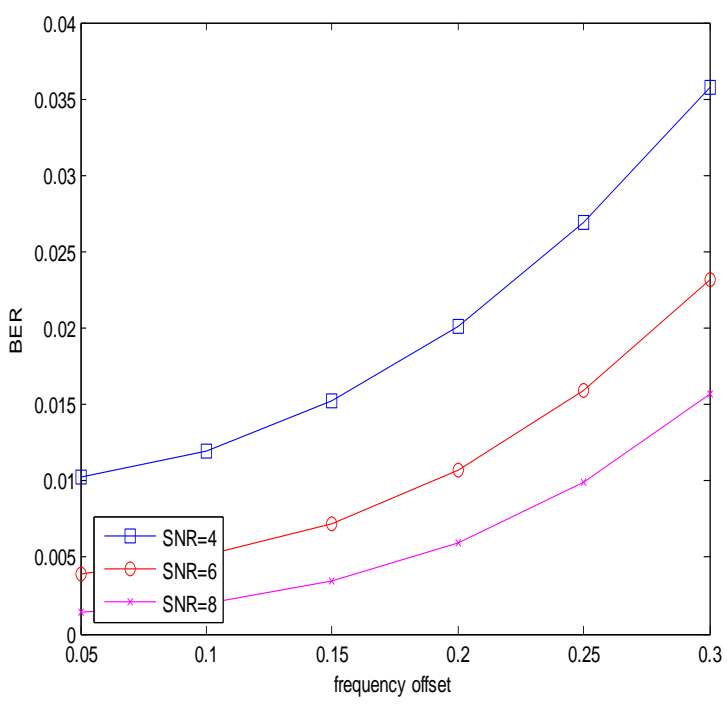

Fig. (3). BER vs. frequency offset for 2 receivers.

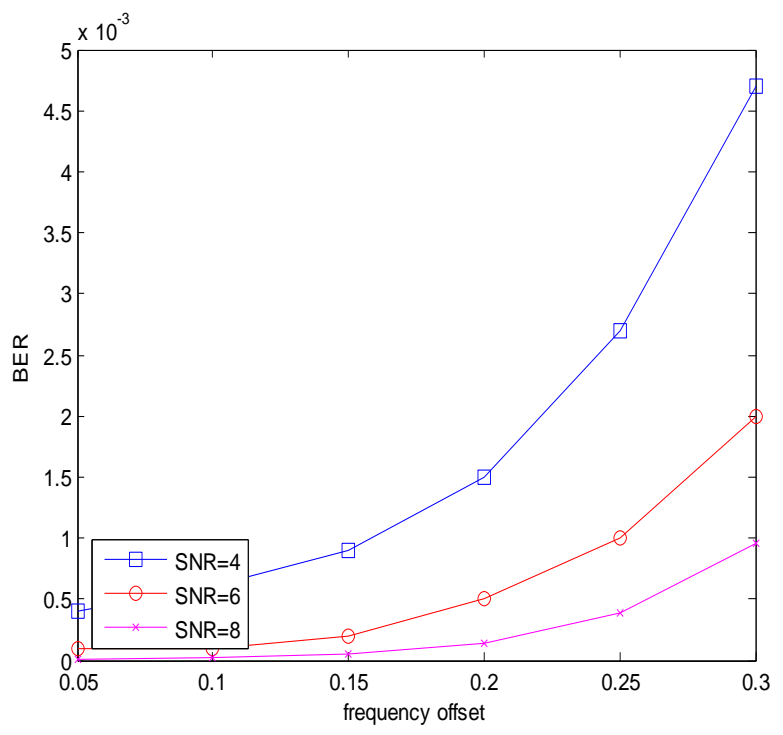

Fig. (4). BER vs. frequency offset for 4 receivers. 


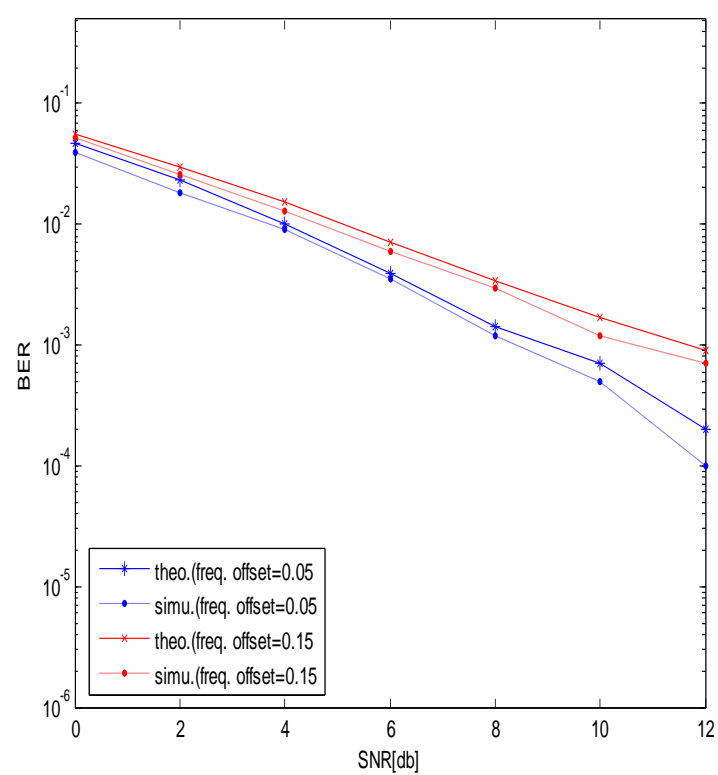

Fig (5).BER vs SNR for freq offset $=0.05,0.15$ and 2 receivers.

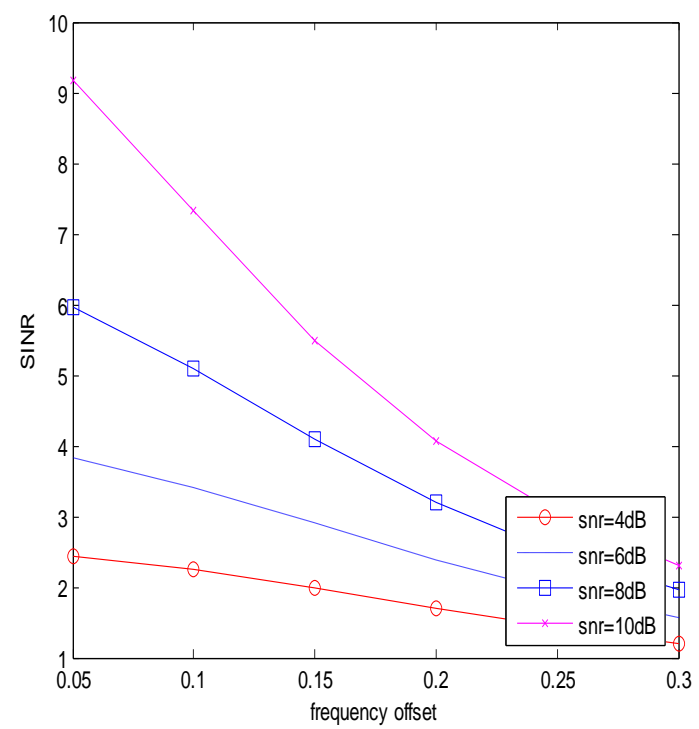

Fig (6).SINR vs frequency offset for different SNR values

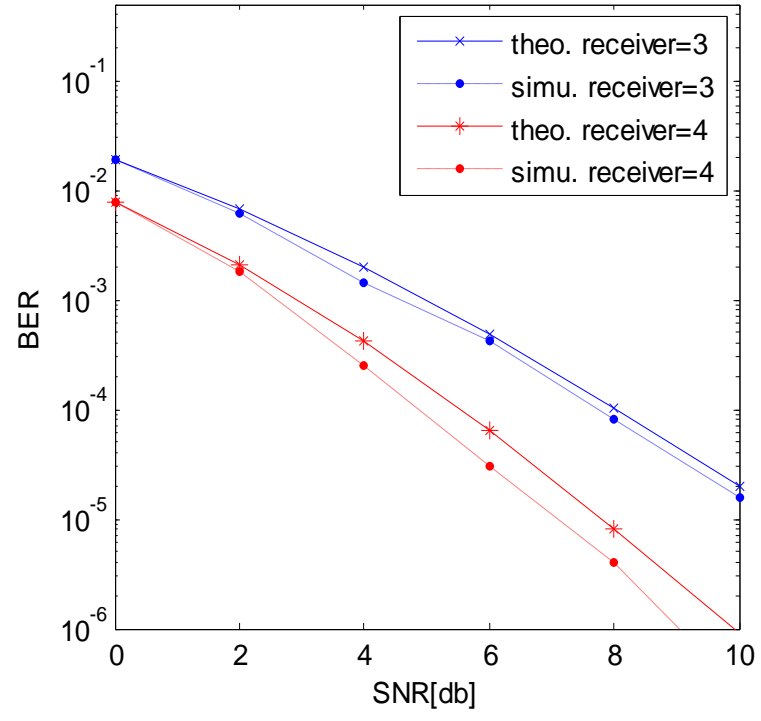

Fig (7).BER vs SNR for frequency offset 0.05 and different values of $\mathrm{m}$.

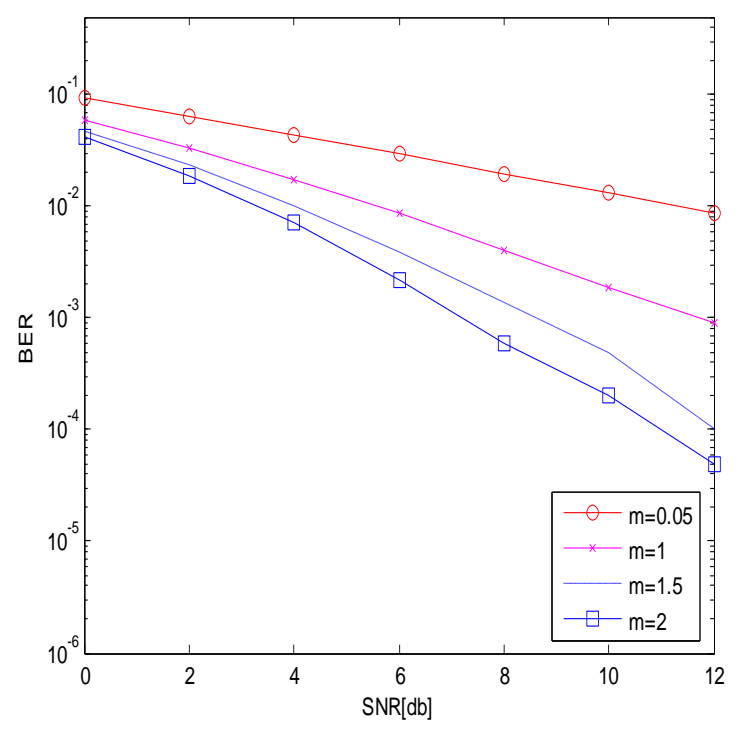

Fig (8).BER vs SNR for freq offset $=0.05$ and $\mathrm{m}=1.5$ and receiver $=3,4$. 


\section{CONCLUSION}

In this paper, we have studied the performance of OFDM signals over nakagami-m channels. For simulation Nakagami fading has been generated by using Rayleigh and ricean fading by the method given in [2].Expression for probability of error is derived. Bit error rate versus SNR, bit error rate versus frequency offset for different number of receivers, bit error rate versus frequency offset for different $\mathrm{m}$, BER for various number of receivers, bit error rate versus frequency offset for different number SNR have been evaluated in this paper. Apart from this signal to interference plus noise ratio is also calculated with respect to different frequency offsets and for different SNR. We observe that the theoretical results match the Monte Carlo simulation results exactly.

\section{REFERENCES}

[1] A. Behravan and T. Eriksson," PAPR and Other Measures for OFDM Systems with Nonlinearity" in wireless personal multimedia communications WPMC, Vol. 1, pp. 149-153, 2002

[2] Li Tang, Zhu Hongbo,"Analysis and Simulation of nakagami fading channel with Matlab,"Asia-Pacific conference on environmental electromagnetic CEEM'2003, China,pp. 490-494.

[3] M. Nakagami, "The m-distribution, a general formula of intensity distribution of rapid fading," in Statistical Methods in Radio Wave Propagation, W. G. Hoffman, Ed, Oxford, England: Pergamum, 1960.
[4] Z. Du , J. Cheng and N.C. Beaulieu," Accurate ErrorRate Performance Analysis of OFDM on FrequencySelective Nakagami-m Fading Channels," IEEE Transactions on communications, Vol. 54, NO. 2, Feb 2006.

[5] Z. Kang, K. Yao, and F. Lorenzelli, "Nakagami-m fading modeling in the frequency domain forOFDMsystem analysis," IEEE Commun. Lett., vol. 7, no. 10, pp. 484486, Oct. 2003.

[6] A. Glavieux, P. Y. Cochet, and A. Picart, "Orthogonal frequency division multiplexing with BFSK modulation in frequency selective Rayleigh and Rician fading channels," IEEE Trans. Commun., vol. 42, no. 2-4, pp. 1919-1928, Feb./Mar./Apr. 1994.

[7] D. Brennan. .Linear diversity combining techniques, Proc. IRE, vol. 47, pp. 1075-1102, June 1959

[8] P. H. Moose," A Technique for Orthogonal Frequency Division Multiplexing Frequency Offset Correction" IEEE Transactions on communications, Vol . 42, No. 10, pp. 2908-2914, Oct 1994.

[9] A. Goldsmith, "Wireless Communications", Cambridge University Press, second edition,2007.

[10] I. S. Gradshteyn and I. M. Ryzhik, Table of Integrals, Series and Products. San Diego, CA: Academic Press, 1995. 